Hamid Taheri • Mohammd Reza R. Moghaddam •

Mohammad Amin Rostamyari

\title{
Some properties on $\mathrm{IA}_{\mathrm{Z}}$-automorphisms of groups
}

Received: 19 September 2018 / Accepted: 7 May 2019 / Published online: 1 June 2019

(C) The Author(s) 2019

\begin{abstract}
Let $G$ be a group and $\operatorname{IA}(G)$ denote the group of all automorphisms of $G$, which induce identity map on the abelianized group $G_{a b}=G / G^{\prime}$. Also the group of those IA-automorphisms which fix the centre element-wise is denoted by $\operatorname{IA}_{Z}(G)$. In the present article, among other results and under some condition we prove that the derived subgroups of finite $p$-groups, for which $\mathrm{IA}_{\mathrm{Z}}$-automorphisms are the same as central automorphisms, are either cyclic or elementary abelian.
\end{abstract}

Mathematics Subject Classification $\quad 20 \mathrm{~F} 28 \cdot 20 \mathrm{~F} 18 \cdot 20 \mathrm{D} 45 \cdot 20 \mathrm{~F} 14$

\section{Introduction and preliminaries}

An automorphism $\alpha$ of a group $G$ is called IA-automorphism if $x^{-1} \alpha(x) \in G^{\prime}$, for all $x \in G$. This concept was introduced by Bachmuch [1] in 1965. We remark the letters I and A as to remind the reader that are those automorphisms which induce identity on the abelianized group, $G / G^{\prime}$.

Also, if $x^{-1} \alpha(x) \in Z(G)$ for all $x \in G$, then we say that $\alpha$ is a central automorphism, and if $\alpha$ preserves all conjugacy classes of $G$, then it is called a class preserving automorphism. The set of all such automorphisms are denoted by $\operatorname{IA}(G), \operatorname{Aut}_{Z}(G)$ and $\operatorname{Aut}_{C}(G)$, respectively. These concepts were introduced and studied by Curran [2] in 2001 and Yadav [15,16] in 2009 and 2013. Clearly, the set of all IA-automorphisms, which fix the centre element-wise, forms a normal subgroup of $\operatorname{IA}(G)$ and is denoted by $\operatorname{IA}_{Z}(G)$ (see $[11,12]$ for more information).

For any element $x$ of a group $G$ and automorphism $\alpha$ of $G$, the autocommutator of $x$ and $\alpha$ is defined as follows:

$$
[x, \alpha]=x^{-1} x^{\alpha}=x^{-1} \alpha(x) .
$$

H. Taheri · M. R. R. Moghaddam

Department of Mathematics, Mashhad Branch, Islamic Azad University, Mashhad, Iran

E-mail: h_taheri2014@yahoo.com

M. R. R. Moghaddam · M. A. Rostamyari

Department of Mathematics, Khayyam University, Mashhad, Iran

E-mail: m.a.rostamyari@khayyam.ac.ir

M. R. R. Moghaddam ( $\triangle)$

Department of Pure Mathematics, Centre of Excellence in Analysis on Algebraic Structures, Ferdowsi University of Mashhad, P.O.BOX 1159, Mashhad 91775, Iran

E-mail: m.r.moghaddam@khayyam.ac.ir; rezam@ferdowsi.um.ac.ir 
Now, using the above notation, we have the following:

$$
\begin{aligned}
\operatorname{Aut}_{\mathrm{Z}}(G) & =\{\alpha \in \operatorname{Aut}(G) \mid[x, \alpha] \in Z(G), \quad \forall x \in G\}, \\
\operatorname{Aut}_{\mathrm{C}}(G) & =\left\{\alpha \in \operatorname{Aut}(G) \mid x^{\alpha} \in x^{G}, \quad \forall x \in G\right\}, \\
\operatorname{IA}_{\mathrm{Z}}(G) & =\left\{\alpha \in \operatorname{Aut}(G) \mid[x, \alpha] \in G^{\prime}, \alpha(z)=z, \quad \forall x \in G \text { and } z \in Z(G)\right\} .
\end{aligned}
$$

One can easily check that any class preserving automorphism is an IA-automorphism, which fixes the centre element-wise and hence

$$
\begin{aligned}
\operatorname{Inn}(G) & \leq \operatorname{Aut}_{\mathrm{C}}(G) \leq \operatorname{IA}_{\mathrm{Z}}(G) \leq \mathrm{IA}(G) \leq \operatorname{Aut}(G), \\
Z(\operatorname{Inn}(G)) & \leq \operatorname{Aut}_{\mathrm{Z}}(G) \leq \operatorname{Aut}(G) .
\end{aligned}
$$

The following example shows that every $\mathrm{IA}_{\mathrm{Z}}$-automorphism is not necessarily inner automorphism.

Example 1.1 Consider the group

$$
G=\left\langle a, b, x \mid[a, x]=[b, x]=1,[a, b]=x^{s}, s \neq 1\right\rangle .
$$

Clearly, $G^{\prime}=\left\langle x^{s}\right\rangle, \quad Z(G)=\langle x\rangle$ and $G / Z(G)=\langle\bar{a}, \bar{b}\rangle \cong \operatorname{Inn}(G)$. The IAZ-automorphism $\alpha$ defined by $\alpha(a)=a x^{s}, \quad \alpha(b)=b x^{s}, \alpha(x)=x$ is a non-inner automorphism.

Note that, $\operatorname{Aut}(G)$ fixes the derived subgroup $G^{\prime}$ element-wise and using this property, we have the following.

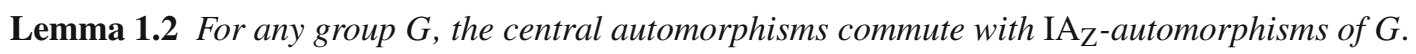

Proof Assume $\alpha$ is a central automorphism of $G$, then $\alpha(x)=x z$ for any $x \in G$ and some element $z \in Z(G)$. Clearly, every central automorphism $\alpha$ fixes $G^{\prime}$ element-wise, and hence for any $\beta \in \operatorname{IAz}_{Z}(G)$, we have

$$
\alpha \beta(x)=\alpha\left(x x^{-1} \beta(x)\right)=\alpha(x) x^{-1} \beta(x)=x z x^{-1} \beta(x)=\beta(\alpha(x))=\beta \alpha(x),
$$

which gives the result.

\section{IA $_{\mathrm{Z}}$-automorphisms of a group}

Considering the converse of Schur's Theorem, Niroomand [10] proved that if the derived subgroup $G^{\prime}$ of a given group $G$ is finite and its central factor group, $G / Z(G)$, is generated by $d$ elements, then $|G / Z(G)| \leqslant\left|G^{\prime}\right|^{d}$. Also, Sury [14] generalized this result as follows.

If $G^{\prime}$ is finite and $G / Z(G)$ is generated by $d$ elements, then $|\operatorname{Inn}(G)| \leqslant|K(G)|^{d}$, where $K(G)=\langle[x, \alpha]|$ $x \in G, \alpha \in \operatorname{Aut}(G)\rangle$ is the autocommutator subgroup of the group $G$, see also [4] and [8].

Here, we give a further generalized version of the above result, which improves [10].

Theorem 2.1 Let $G$ be any group with finite derived subgroup. If $d$ is the minimal number of generators of the central factor group of $G$, then $\left|\mathrm{IA}_{\mathrm{Z}}(G)\right| \leqslant\left|G^{\prime}\right|^{d}$.

Proof Assume that $G / Z(G)$ has a minimal set of generators $x_{1} Z(G), \ldots, x_{d} Z(G)$ and $\alpha \in \operatorname{IA}_{Z}(G)$, which fixes $Z(G)$ element-wise. Consider the following map:

$$
\psi: \operatorname{IA}_{Z}(G) \longrightarrow \underbrace{G^{\prime} \times G^{\prime} \times \cdots \times G^{\prime}}_{d-\text { times }}
$$

given by $\psi(\alpha)=\left(\left[x_{1}, \alpha\right],\left[x_{2}, \alpha\right], \cdots,\left[x_{d}, \alpha\right]\right)$, for all $\alpha \in \operatorname{IA}_{Z}(G)$.

One can easily check that $\psi$ is injective, as $\psi(\alpha)=\psi(\beta)$ implies that $\left[x_{i}, \alpha\right]=\left[x_{i}, \beta\right]$, for all $1 \leqslant i \leqslant d$ and any $\alpha, \beta \in \operatorname{IA}_{\mathrm{Z}}(G)$. Hence, $\alpha=\beta$ and so $\left|\operatorname{IA}_{\mathrm{Z}}(G)\right| \leqslant\left|G^{\prime}\right|^{d}$.

Remark 2.2 One notes that the above theorem improves the result in $[10]$, as $\operatorname{Inn}(G) \leq \operatorname{Aut}_{\mathrm{C}}(G) \leq \operatorname{IA}_{\mathrm{Z}}(G)$.

Clearly, Autz $(G) \cap \operatorname{Inn}(G)=Z(\operatorname{Inn}(G))$, for any group $G$. Now, we use this property to obtain the following. 
Lemma 2.3 Let $G$ be a group such that $Z(G) \leq G^{\prime}$. Then $\operatorname{Aut}_{\mathrm{Z}}(G)=\operatorname{IA}_{\mathrm{Z}}(G)$ if and only if $\operatorname{Inn}(G)=$ $Z(\operatorname{Inn}(G))$.

Proof Assume $\operatorname{Aut}_{\mathrm{Z}}(G)=\operatorname{IA}_{\mathrm{Z}}(G)$, then $\operatorname{Inn}(G)=\operatorname{IA}_{\mathrm{Z}}(G) \cap \operatorname{Inn}(G)=Z(\operatorname{Inn}(G))$.

Conversely, let $\operatorname{Inn}(G)=Z(\operatorname{Inn}(G))$. Then $G^{\prime} \leq Z(G)$ and so the definition of $\operatorname{IA}_{Z}(G)$ implies that $\operatorname{IA}_{\mathrm{Z}}(G) \leq \operatorname{Aut}_{\mathrm{Z}}(G)$. On the other hand, by the assumption $\operatorname{Aut}_{\mathrm{Z}}(G) \leq \operatorname{IA}_{\mathrm{Z}}(G)$. Therefore, $\operatorname{Aut}_{\mathrm{Z}}(G)=$ $\operatorname{IA}_{Z}(G)$.

The next result shows that the subgroup $Z\left(\operatorname{IA}_{\mathrm{Z}}(G)\right)$ is between $Z(\operatorname{Inn}(G))$ and $\operatorname{Aut}_{\mathrm{Z}}(G)$.

Proposition 2.4 For any group $G$,

$$
\operatorname{Aut}_{\mathrm{Z}}(G) \cap \mathrm{IA}_{\mathrm{Z}}(G)=Z\left(\mathrm{IA}_{\mathrm{Z}}(G)\right) .
$$

Proof Suppose that $\alpha \in \operatorname{Aut}_{\mathrm{Z}}(G) \cap \mathrm{IA}_{\mathrm{Z}}(G)$ and $\beta \in \mathrm{IA}_{\mathrm{Z}}(G)$. Lemma 1.2 implies that $[\alpha, \beta]=i d$., as $\alpha \in \operatorname{Aut}_{\mathrm{Z}}(G)$ and hence $\alpha \in Z\left(\operatorname{IA}_{\mathrm{Z}}(G)\right)$.

Conversely, for any $\alpha \in Z\left(\operatorname{IA}_{\mathrm{Z}}(G)\right)$ and $\beta \in \operatorname{Inn}(G) \leq \operatorname{IA}_{\mathrm{Z}}(G)$, we have $[\alpha, \beta]=i d$. This implies that $\alpha \in C_{\text {Aut }(G)}(\operatorname{Inn}(G))=\operatorname{Aut}_{\mathrm{Z}}(G)$, and so $\alpha \in \operatorname{Aut}_{\mathrm{Z}}(G) \cap \operatorname{IA}_{\mathrm{Z}}(G)$.

In 1940, Hall [3] introduced the concept of isoclinism between two groups and it was extended to $n$ isoclinism, which is an equivalent relation among all groups and it is weaker than isomorphism. In 1976, Leedham-Green and McKay [7] extended this concept to isologism with respect to a given variety of groups. There have been extensive studies in this area of mathematics (see [5,6], for more information).

Definition 2.5 Let $G$ and $H$ be arbitrary groups and assume $\alpha: G / Z(G) \rightarrow H / Z(H)$ and $\beta: G^{\prime} \rightarrow H^{\prime}$ be isomorphisms such that the following diagram is commutative:

$$
\begin{array}{rll}
\frac{G}{Z(G)} \times \frac{G}{Z(G)} & \stackrel{\alpha \times \alpha}{\longrightarrow} & \frac{H}{Z(H)} \times \frac{H}{Z(H)} \\
f_{1} \downarrow & & \downarrow f_{2} \\
G^{\prime} & \stackrel{\beta}{\longrightarrow} & H^{\prime},
\end{array}
$$

where $f_{1}\left(g_{1} Z(G), g_{2} Z(G)\right)=\left[g_{1}, g_{2}\right]$ and $f_{2}\left(h_{1} Z(H), h_{2} Z(H)\right)=\left[h_{1}, h_{2}\right]$, for each $h_{i} \in \alpha\left(g_{i} Z(G)\right)$, $i=1,2$, and $\beta$ is an isomorphism induced by $\alpha$. Then $(\alpha, \beta)$ is said to be isoclinism, so that $G$ and $H$ are called isoclinic and denoted by $G \sim H$.

Here, we characterize all finite $p$-groups, in which their IA $\mathrm{Z}$-automorphisms are equal to central automorphisms. Yadav [16] proved that if two finite groups $G$ and $H$ are isoclinic, then $\operatorname{Aut}_{C}(G) \cong \operatorname{Aut}_{C}(H)$. With the same assumption, Pradeep [11] showed that $\operatorname{IA}_{Z}(G) \cong \mathrm{IA}_{Z}(H)$ and he applied his result to prove the following.

Theorem 2.6 ([11], Theorem $\mathrm{B}(1))$ Let $G$ be a finite p-group. Then $\operatorname{Aut}_{\mathrm{Z}}(G)=\operatorname{IA}_{\mathrm{Z}}(G)$ if and only if $Z(G)=G^{\prime}$.

The following results can be deduced using the above theorem.

Proposition 2.7 Let $G$ be a d-generating finite p-group and $\operatorname{Aut}_{\mathrm{Z}}(G)=\operatorname{IA}_{\mathrm{Z}}(G)$. Then $\left|\operatorname{IA}_{\mathrm{Z}}(G)\right|=\left|G^{\prime}\right|^{d}$.

Proof The assumption and Theorem 2.6 imply that $G / Z(G)$ is abelian. Hence, assume $G / Z(G)=\left\langle\overline{x_{1}}\right\rangle \times$ $\left\langle\overline{x_{2}}\right\rangle \times \cdots \times\left\langle\overline{x_{d}}\right\rangle$, where $\overline{x_{i}}=x_{i} Z(G)$ is of order $p^{n_{i}}$, for some positive integer $n_{i}, i=1, \ldots, d$. Since $Z(G)=G^{\prime} \leq \Phi(G)$, by $[16]$ Lemma $3.5,\left\{x_{1}, \ldots, x_{d}\right\}$ is the minimal generating set for $G$. On the other hand, $\operatorname{Hom}\left(\left\langle\overline{x_{i}}\right\rangle, G^{\prime}\right)=G^{\prime}$, and hence Corollary $2.2[11]$ implies that

$$
\left|\mathrm{IA}_{\mathrm{Z}}(G)\right|=\left|\operatorname{Hom}\left(G / Z(G), G^{\prime}\right)\right|=\Pi_{i=1}^{d}\left|\operatorname{Hom}\left(\left\langle\bar{x}_{i}\right\rangle, G^{\prime}\right)\right|=\Pi_{i=1}^{d}\left|G^{\prime}\right|=\left|G^{\prime}\right|^{d} .
$$

Theorem 2.8 Let $G$ and $H$ be isoclinic finite p-groups, and $\operatorname{Aut}_{\mathrm{Z}}(G)=\operatorname{IA}_{\mathrm{Z}}(G)$. Then $\operatorname{Aut}_{\mathrm{Z}}(H)=\operatorname{IA}_{\mathrm{Z}}(H)$ if and only if $|G|=|H|$. 
Proof Assume that $\operatorname{Aut}_{\mathrm{Z}}(H)=\mathrm{IA}_{\mathrm{Z}}(H)$. The assumption and Theorem 2.6 imply that $G^{\prime}=Z(G)$. The isoclinic property of $G$ and $H$ implies that

$$
|Z(G)|=\left|G^{\prime}\right|=\left|H^{\prime}\right|=|Z(H)| .
$$

Therefore,

$$
|H|=|H / Z(H)||Z(H)|=|G / Z(G)||Z(G)|=|G| .
$$

Conversely, if $|G|=|H|$ then $|Z(G)|=|Z(H)|$, as $G$ and $H$ are isoclinic. On the other hand, $Z(G)=$ $G^{\prime} \cong H^{\prime}$ and hence $\left|H^{\prime}\right|=|Z(H)|$. Clearly, $G$ is nilpotent of class 2 and isoclinic with $H$, which imply that $H$ is also nilpotent of class 2. Then $H^{\prime} \leq Z(H)$ and so $H^{\prime}=Z(H)$. Now, Theorem 2.6 implies that $\operatorname{Aut}_{\mathrm{Z}}(H)=\operatorname{IA}_{\mathrm{Z}}(H)$.

The following example gives a class of isoclinic groups, in which their $\mathrm{IA}_{\mathrm{Z}}$-automorphisms are the same as central automorphisms.

Example 2.9 Consider the group

$$
\begin{aligned}
G_{i}=\left\langle a, a_{1}, a_{2}, \cdots, a_{2 s}\right| a^{p^{r+t}}=1, a_{1}^{p^{r}}=\cdots & =a_{2 s}^{p^{r}}=a^{p^{i}}, \\
{\left[a_{1}, a_{2}\right]=\left[a_{2}, a_{3}\right] } & \left.=\cdots=\left[a_{2 s-1}, a_{2 s}\right]=a^{p^{t}}\right\rangle,
\end{aligned}
$$

where $r, s, t \geqslant 1$ and $1 \leqslant i \leqslant r$.

Clearly, the group $G_{i}$ is nilpotent of class 2 and it is of order $p^{r(2 s+1)+t}$. Also one can easily see that

$$
Z\left(G_{i}\right) \cong C_{p^{r+t}}, \quad G_{i}^{\prime} \cong C_{p^{r}}, \quad \frac{G_{i}}{Z\left(G_{i}\right)} \cong\left(C_{p^{r}}\right)^{2 s} .
$$

Now, Proposition 3.2 of [13] and Lemma 2.3 imply that

$$
\operatorname{Aut}_{\mathrm{Z}}\left(G_{i}\right)=\mathrm{IA}_{\mathrm{Z}}\left(G_{i}\right)=Z\left(\operatorname{Inn}\left(G_{i}\right)\right)=\operatorname{Inn}\left(G_{i}\right) \cong\left(C_{p^{r}}\right)^{2 s},
$$

as the derived subgroup of the group $G_{i}$ is cyclic, for all $1 \leqslant i \leqslant r$.

The following lemma of Morigi [9] is useful for our further investigation.

Lemma 2.10 ([9], Lemma 0.4) Let $G$ be a finite nilpotent group of class 2. Then $\exp \left(G^{\prime}\right)=\exp (G / Z(G))$ and in the decomposition of $G / Z(G)$ into direct product of cyclic groups at least two factors of maximal orders must occur.

We recall that the centre of any non-abelian $p$-group of order $p^{n}$ lies between $p^{2}$ and $p^{n-2}$.

Theorem 2.11 Let $G$ be a non-abelian p-group with $\operatorname{Aut}_{\mathrm{Z}}(G)=\operatorname{IA}_{\mathrm{Z}}(G)$. If $p^{3} \leq|G| \leq p^{7}$, then the derived subgroups of such groups are either cyclic or elementary abelian p-groups.

Proof By the assumption and Theorem 2.6, $Z(G)=G^{\prime}$. It is sufficient to show that $Z(G)$ is cyclic or elementary abelian. The result is obvious, when $G$ is of order $p^{3}$ or $p^{4}$. Now assume $G$ is of order $p^{5}$, then $|Z(G)|=p^{2}$ or $p^{3}$. If $|Z(G)|=p^{3}$, then $|G / Z(G)|=p^{2}$. By Morigi's Lemma, we have

$$
\exp (Z(G))=\exp \left(G^{\prime}\right)=\exp (G / Z(G))=p .
$$

Hence, $Z(G)$ is elementary abelian. Suppose that $|G|=p^{6}$. If $|Z(G)|=p^{2}$ or $p^{4}$, then Morigi's Lemma gives the result. Assume $|Z(G)|=p^{3}$ and $Z(G) \cong C_{p^{2}} \times C_{p}$. Then

$$
\exp (Z(G))=\exp \left(G^{\prime}\right)=\exp (G / Z(G))=p^{2} .
$$

Therefore,

$$
G / Z(G) \cong C_{p^{2}} \times C_{p},
$$

which contradicts Morigi's Lemma and so $Z(G) \cong C_{p^{3}}$ or $C_{p} \times C_{p} \times C_{p}$. 
Finally, assume $|G|=p^{7}$. If $|Z(G)|=p^{2}$ or $p^{5}$, then again the result is obtained, by Morigi's Lemma. Assume $Z(G)$ is of order $p^{3}$ and $Z(G) \cong C_{p^{2}} \times C_{p}$, then

$$
G / Z(G) \cong C_{p^{2}} \times C_{p^{2}} .
$$

So $G$ has two non-commutative generators and hence $G^{\prime}$ is cyclic, which contradicts the property that $Z(G)=$ $G^{\prime}$.

If $|Z(G)|=p^{4}$ and

$$
Z(G) \cong C_{p^{2}} \times C_{p} \times C_{p}, C_{p^{3}} \times C_{p} \text { or } C_{p^{2}} \times C_{p^{2}}
$$

then

$$
\exp (Z(G))=\exp \left(G^{\prime}\right)=\exp (G / Z(G))=p^{2} \text { or } p^{3}
$$

and $|G / Z(G)|=p^{3}$, which contradicts Morigi's Lemma and so,

$$
Z(G) \cong C_{p^{4}} \text { or } \quad C_{p} \times C_{p} \times C_{p} \times C_{p} .
$$

Acknowledgements The authors would like to thank the referees for their helpful suggestions, which made the article more readable.

Open Access This article is distributed under the terms of the Creative Commons Attribution 4.0 International License (http:// creativecommons.org/licenses/by/4.0/), which permits unrestricted use, distribution, and reproduction in any medium, provided you give appropriate credit to the original author(s) and the source, provide a link to the Creative Commons license, and indicate if changes were made.

\section{References}

1. Bachmuth, S.: Automorphisms of free metabelian groups. Trans. Am. Math. Soc. 118, 93-104 (1965)

2. Curran, M.J.; McCughan, D.J.: Central automorphisms that are almost inner. Commun. Algebra 29, 2081-2087 (2001)

3. Hall, P.: The classification of prime-power groups. J. Reine Angew. Math. 182, 130-141 (1940)

4. Hegarty, P.V.: The absolute centre of a group. J. Algebra 169, 929-935 (1994)

5. Hekster, N.S.: On the structure of $n$-isoclinism classes of groups. J. Pure Appl. Algebra. 40, 63-85 (1986)

6. Hekster, N.S.: Varieties of groups and isologism. J. Aust. Math. Soc. Ser. A 46, 22-60 (1989)

7. Leedham-Green, C.R.; Mckay, S.: Baer-invariants, isologism, varietal laws and homology. Acta Math. 137, 99-150 (1976)

8. Moghaddam, M.R.R.; Parvaneh, F.; Naghshineh, M.: The lower autocenteral series of abelian group. Bull. Korean Math. Soc. 48(1), 79-83 (2011)

9. Morigi, M.: On the minimal number of generators of finite non-abelian $p$-groups having an abelian automorphism group. Commun. Algebra 23(6), 2045-2065 (1995)

10. Niroomand, P.: The converse of Schur's Theorem. Arch. Math. 94, 401-403 (2010)

11. Pradeep, K.R.: On IA-automorphisms that fix the centre element-wise. Proc. Indian Acad. Sci. Math. Sci. 124(2), 169-173 (2014)

12. Singh, S.; Gumber, D.; Kalra, H.: IA-automorphisms of finitely generated nilpotent groups. J. Algebra 19(7), 271-275 (2014)

13. Soleimani, R.: On some $p$-subgroups of automorphism group of a finite $p$-groups. Vietnam J. Math. 36(1), 63-69 (2008)

14. Sury, B.: A generalization of a converse of Schur's Theorem. Arch. Math. 95, 317-318 (2010)

15. Yadav, M.K.: On central automorphisms fixing the centre element-wise. Commun. Algebra 37, 4325-4331 (2009)

16. Yadav, M.K.: On finite $p$-groups whose central automorphisms are all class preserving. Commun. Algebra 41(12), 4576-4592 (2013)

Publisher's Note Springer Nature remains neutral with regard to jurisdictional claims in published maps and institutional affiliations. 